unter der Benennung C.linifolia mit C.Scheuchzeri Vill, als Varietät, und C.pubescens $S \mathrm{eh}$ m. - Ves $\downarrow$ macht aus den erwähnten Formen in seinem für die damalige Zeit sehr brauchbaren Manuale botanicum (Klagenf. 1805) vier verschiedene Arten: C. rotundifolia, pusilla, linifolia, cespitosa, welche analytisch auf folgende Weise sich unterscheiden lassen:

1. Behaart: C. linifolia.

Kahi - 2.

2. Die oberen Blälter ganzrandig : rotundifolia.

Alle Blätter gesägl -3 .

3. Corolle allmälig sich erweiternd: pusilla.

» bauchig: cespitosa.

(Fortsetzung folgt.)

\title{
Einige wohlgemeinte Worte
}

über botanische Excursionen und Sammlungen.

Von P. M. Opiz, in Prag.

CVorgelesen in der Section für Naturwissenschaft und Mathematik bei der k. böhm. Gesellschaft der Wissenschaften am 5. April 1852. *)

Seit dem Jahre 1800 habe ich mich mit reger Liebe der Pflanzenkunde, unter nichts weniger als begünstigenden Umständen ergeben, und daher vielfache Hindernisse und Schwierigkeiten kennen gelernt, die Andern ganz unbekannt geblieben sein dürften. Seit dem Jahre 1818, wo ich, aus eben diesem Anlasse, die e r s le Pflanzentausch-Anstalt in Prag zur Behebung mancher dieser Schwierigkeiten errichtete, habe ich diese nun durch 34 Jahre mit gleich regem Eifer fortgeführt, und will, so Gott will, die noch kurze Zeit meines irdischen Hierseins noch dazu benützen, das Angefangene, so lange es geht, fortzusetzen. Bei den mancherlei Erfahrungen, die ich im Verlaufe von mehr als einem halben Jahrhundert gemacht habe, dürfte es wohl nicht ganz übcrflüssig und nutzlos erscheinen, über obige zwei, für den Botaniker gewiss wichtige Gegenstände, in den gegenwärtigen geschätzten Blättern einige wohlgemeinte Worte zu sagen.

Bereits sind schon mehr als 100.000 Pflanzenarten bekannt, und doch sieht man noch immer den grössten Theil der Sammlungen, selbst öffentlicher Anstalten, im Vergleiche zu dieser Masse, so artenarm, dass sich uns die Frage unwilikürlich aufdrängt, woher dies komme? Leicht erklärlich wird es jedoch, wenn man

*) Herr 0 p 1 z war so gütig uns den vollen Inhalt seiner Vorlesung mitzutheilen, allein so interessant auch der ganze Aufsatz ist, so könuen wir ihn unsern Lesern doch nur auszugsweise vorführen, da derselbe uns sonst (er umlasst 3 angeschriebene Foliobögen) zu viel Raum unseres engbegränzten Blattes kosten würde. Wir sehen uns daher genöthiget, nur den Inhalt des ersten Bogens theilweise zu benützen und den der zwei andern, welche von den botanischen Excursionen, dem Einlegen der Pllanzen und der geachteten Tauschanstalt des Herrn 0 p i z handeln, vielleicht einer spälern Zeit zu überlassen, in der wir mit Original-Aufsätzen und wichtigen Mittheilungen minder versehen sind, als eben jetzt.

A n m. d. Re d. 
bedenkt, wie wenig Verkehr noch bis jetzt zwischen den Botanikern derg a $\mathrm{n} z$ e $\mathrm{n}$ W e it bestand, - wie bald der in Wissen weiter Vorgerückte aufhört auch für Andere zu sammeln, und wie die meisten Sammler, meist jüngere Männer, noch viel $\mathrm{zu}$ wenig das minder $\mathrm{Be}$ kannte oder noch. Unbekannle beim Sammeln beachten, - wie so selten öffentliche Sammlungen auch so angelegt und eingerichtet sind, dass sie stets zugänglich und belehrend erscheinen, wie noch seltener sich die Priester Florens dem Laien mittheilend zeigen, und ihre Sammlungen seinen wissbegierigen Augen öffnen. Dass sie ihm in Bestimmung zweifelhafter und schwieriger Pflanzenarten behilflich sein sollten, darf man von ihnen gar nicht fordern, und eine Anstalt, von der man diese Bestimmung $z u$ erwarten hoffen dürte, ist meines Wissens nicht vorhanden, allein wahres und dringendes Bedürfniss. Die meisten Sammlungen sind auch so wenig instructiv, dass man in ihnen selten die wahre Belehrung, dagegen aber sehr viel des noch Unbestimmten und noch nicht gehörig critisch Gesichteten findet. Selbst die Einrichtung der meisten naturhistorischen Vereine und Gesellschaften ist so beschaffen, dass siè dem angehenden Freunde über Wissenschaft oft keinen Zutritt gestatten, und im bestem Falle, ihm wenig Aufmunterung und Nutzen gewähren, und beides ist doch nothwendig, um gerade dem Jünger Lust und Liebe zum Wissen zu wecken, zu nähren und auszubilden, um an ihm einen treuen und eifrigen Anhänger der Wissenschaft zu gewinnen. Man darf auf den Anfänger keineswegs geringschätzend herabblicken, denn er hat die Vorarbeiten seiner Vorgänger vor sich, hat schärfere Sinne, und die ihm innewohnende ungeschwächte, frische Lebenskraft, kann daher bei regem Eifer, Fleiss und günstiger Gelegenheit seine Vorgänger in vielen Fällen sehr leicht überholen, wenn diese nicht mit der Zeit gleichen Schritt halten.

Nach den mir bekannt gewordenen Beschreibungen hat noch das $W$ i 1 I d e n o w'sche Herbar in Berlin die zweckmässigste Einrichtung, doch auch hier, wie uns ein Referat des seligen Professors ' $\mathrm{T}$ a u s ch in der "Flora" oder" ,botanischen Zeitung " berichtet, ist des nicht richtig Bestimmten eine schöne Anzahl vorhanden. Ein Hauptfehler, selbst bei derlei Sammlungen, ist die Unvollständigkeit der einzelnen Exemplare. Jede Pflanze sollte man in allen Vegetationszuständen gesammelt haben; jedes Exemplar sollte aber auch stets mit der Original-Etiquelte des Sammlers versehen sein, auf welcher der Fundort, die Zeit des Fundes, nebst dem Namen des Finders stets bemerkt sein müsste, damit man über die Abkunft desselben $n$ ie in $Z$ weifel gerathen könnte; diess ist $\mathrm{nm}$ so nothwendiger, wenn man in die Sammlung die Belege für Systeme, Monographien, Topographien und pflanzengeographische Arbeiten niederlegen will.

Bei jeder Sammlung sollte noch abgesondert eine a t $t$ th $\mathrm{n} t \mathrm{i}-$ sche Sa m m lung errichtet werden, in welche nur jene Pflanzen aufzunehmen wären, welche von den Entdeckern selbst als identisch mit den von ihnen entdeckten Pflanzen erklärt worden sind. 
Manche lieben es, recht viel des Seltenen zu besitzen, und theilen daher aus diesem Grundsatze Niemanden etwas von ihren Schätzen mit, damit nur sie allein im Besitze derselben bleiben möchten. Mich dagegen freuet eine Pflanze nie so sehr, wenn ich sie nicht auch recht Vielen mittheilen kann, und dieser innere Drang nach Mittheilung. war es auch hauptsächlich, der mich im Jahre 1818 zur Gründung meiner Pflanzentausch - Anstalt bewog, welche die erste dieser Art war, und Anlass gegeben hat, dass dermals bereits mehrere dieser Anstalten zum Nutzen und Frommen der Wissenschaft in mehreren Ländern Europa's erstanden sind, deren Hauptfehler aber noch immer darin besteht, dass sie nicht auch unter einander selbst in einen gegenseitigen Verkehr treten.

Je Mehrere sich nun der Wissenschaft ergeben, um so mehr kann diese erweitert, vervollkommet und verbreitet werden; um so mehr Absatz erlangen dann die botanischen Schriften; bei grösserem Absatz können diese sodann auch wohlfeiler geliefert, milhin auch allgemeiner zugänglich werden, und sodann unsere Scientia amabilis, wie sie Vater $L$ in $n$ é mit vollem Rechte nannte, auch immer mehr ins practische Leben übergéhen und der Gesellschaft auch vielfache materielle Vortheile bieten, und unseren Geist zur Bewunderung und Anbetung Gottes erheben und hierdurch unseren moralischen Werth erhöhen.

Bei den botanischen Lehranstalten sollte man die Hörer vorzüglich anleiten:

a) wie sie ihre botanischen Ausflüge einzurichten hälten, um Vieles und Brauchbares einzusammeln;

b) auf welche Art sie die gesammelten Gewächse am leichtesten und richtigsten bestimmen könnten;

c) wie sie die gesammellen Gegenstände am besten zu trocknen und zuzubereiten im Stande wären;

d) welche zweckmässigste Einrichtung sie ihren Sammlungen zu geben hälten und

e) auf welche Art sie selbst die bestehenden PflanzentáuschAnstalten zu ihrem grösstmöglichsten Vortheile, um Zeit und Geld zu ersparen, benützen könnten.

\section{Personalmotizen.}

- Professor Me ttenius, in Freiburg, hat einen Ruf an die Universität Leipzig erhalten und angenommen, wird jedoch die Sommervorlesungen in Freiburg noch abhalten.

- Dr. Sang u in e $t$ ti hat die Professur der Bolanik an der Sapienza zu Rom und die Direclion des botanischen Gartens daselbst erhalten.

- Dr. Heinrich M. W ill k o m m hat sich an der Universität Leipzig zum Privatdocenten habilitirt.

- In der vom Herrn Garteninspector Ed. 0 t t o herausgegehenen Hamburger Garten- und Blumenzeitung Bd. VIII., S. 193. ff. findet sich, in Folge einer im "Phytologist" enthaltenen und hier in Uebersetzung mitgetheilten Darstellung der Verhältnisse N e e s v o n 